\title{
Indentation Methods for Characterising the Mechanical and Fracture Behaviour of Polymer Nanocomposites
}

\author{
Ralf Lach ${ }^{1,2}$, Gyeong-Man Kim³ , Rameshwar Adhikari, Goerg H. Michler5 and Wolfgang \\ Grellmann² \\ ${ }^{1}$ Institut für Polymerwerkstoffe e.V., Institute associated with the University \\ of Applied Science Merseburg, \\ Merseburg, Germany \\ ${ }^{2}$ Martin-Luther University Halle-Wittenberg, Centre of Engineering \\ Halle/Saale, Germany \\ ${ }^{3}$ Centro de Estudios e Investigaciones Tecnicas (CEIT), Department of Biomedical Engineering, \\ San Sebastian (Spain) \\ ${ }^{4}$ Nepal Polymer Institute (NPI) \\ Kathmandu \\ ${ }^{5}$ Martin-Luther University Halle-Wittenberg, Institute of Physics, Halle/Saale, Germany \\ e-mail: ralf.lach@psm.uni-halle.de
}

\begin{abstract}
Morphological analysis of nanocomposites of polyethylene glycol (PEG) based polyurethanes (TPU) and polyhedral oligomeric silsesquioxanes (POSS) were performed using different techniques (transmission electron microscopy, small and wide X-ray scattering, differential scanning calorimetry) as a function of molecular weight of PEG and the PEG/POSS mole ratio. A strong interdependence in crystallisation behaviour between the two crystals species, i.e. the POSS nanocrystals about $5 \mathrm{~nm}$ in size and the crystals in the semicrystalline soft phase of TPU, were found. The mechanical properties (Vickers hardness under load and elastic modulus) determined using recording microindentation techniques at room temperature were significantly improved by POSS for two material formulations. Based on the results of polymethylmethacrylate (PMMA)/silica $\left(\mathrm{SiO}_{2}\right)$ nanocomposites containing up to 20 wt.-\% well-dispersed spherical silica nanoparticles, it has been shown that the indentation fracture mechanics is a straightforward, costand time-effective, powerful tool to analyse the fracture resistance for the novel polymer materials, such as brittle nanostructured polymer-ceramic hybrids.
\end{abstract}

Key word: fracture mechanics, hardness, indentation methods, polymer nanocomposite

\section{Introduction}

Hardness testing methods have become one of the most popular tools in mechanical testing because of their relatively straightforward, fast and sufficiently repeatable performance. Besides, hardly any timeconsuming specimen preparation is required. Another advantage is that the indenter only generates a very small impression due to its small indentation depth. Consequently, the hardness testing works generally in a quasi non-destructive manner, which provides the opportunity to test very small components and thin layers, for which only little information about the mechanical properties can be obtained otherwise. It has to been emphasised, furthermore, that statistically firm correlations between hardness and other mechanical properties, such as the yield stress or the abrasion resistance, exist, at least within one and the same materials group (Grellmann \& Seidler 2007, Herrmann 2007, Balta et al. 2000, Koch 2005).

For the currently used standardised hardness testing procedures (Table 1), a hard indenter is often pressed in the surface of the sample. As a result, a triaxial 
(hydrostatic) state of stress is formed inside the sample below the indenter. These testing methods, which are each suitable for specified materials groups and fields of application, differ, in principle, in the form of the indenter (pyramid, cone, ball, etc.), the material of the indenter (steel, hard metal, diamond), the level of loading (up to $30 \mathrm{kN}$; macro, low-load, micro and nano range), the type of loading (slow = static, impact-like = dynamic) and the method of measuring (under load, after unloading) from each other. The hardness values obtained, which are strongly dependent on the testing procedure and conditions (these information has to be specified in the testing protocol), cannot or only very hardly be converted into each other with just a few exceptions. However, recent trend of materials testing based on a small number of universal methods can be recognized (Grellmann \& Seidler 2007, Herrmann 2007, Balta et al. Calleja \& Fakirov 2000, Koch 2005, VDI/VDE 2000a, VDI/VDE 2000b).

Table 1. Hardness testing methods.

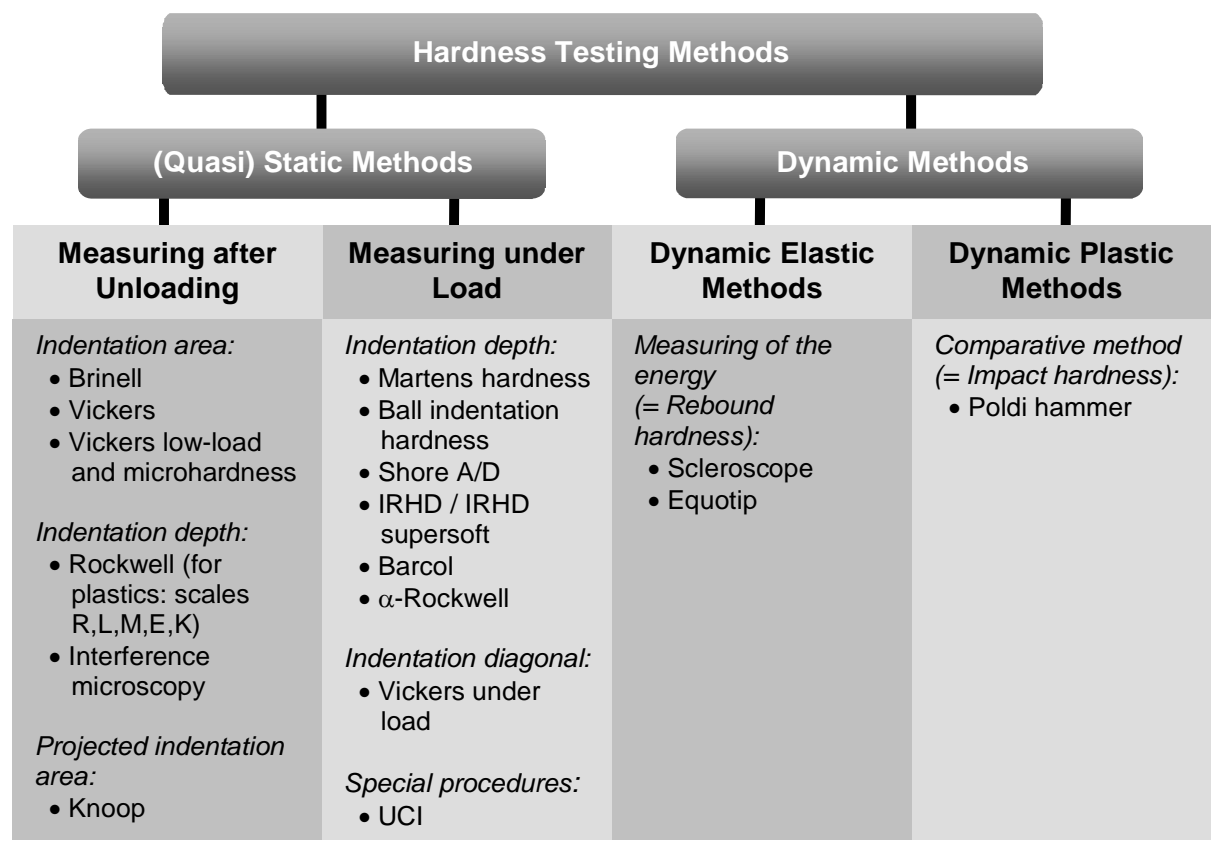

Table 2 Infhencing factos on hardness parameters.

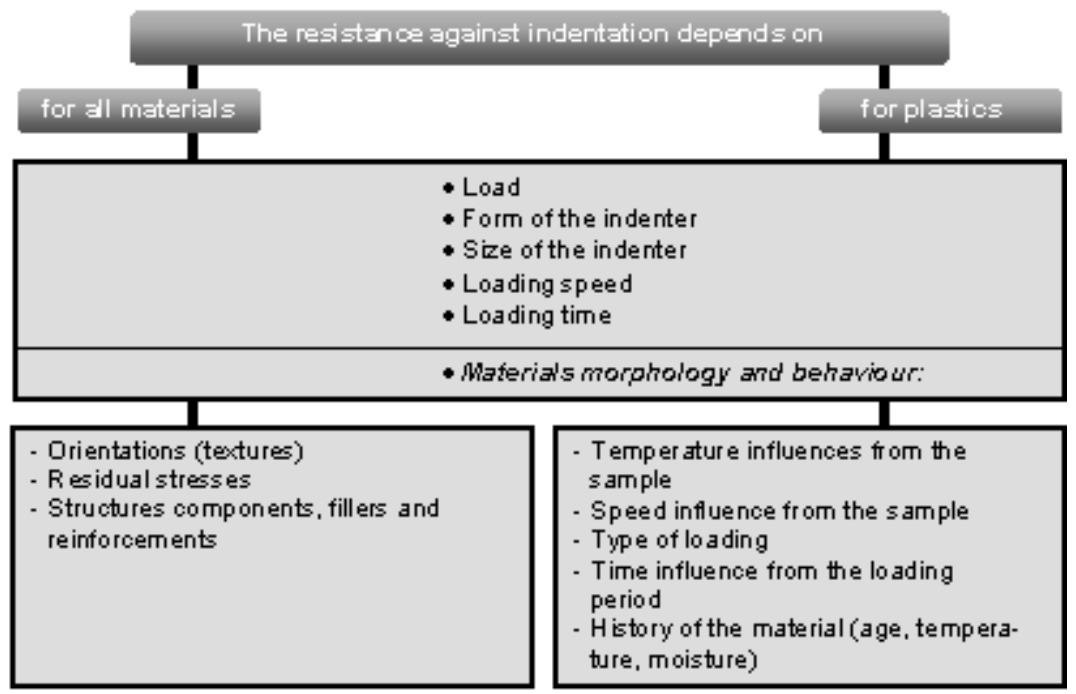


Historically, hardness testing methods were first developed for metallic materials, especially steels (Herrmann 2007, Balta VDI/VDE 2000a). However, hardness testing of plastics is based on many approaches underlying these methods considering the polymer-specific material behaviour (Grellmann \& Seidler 2007, Balta et al. 2000, Koch 2005, VDI/VDE 2000a, VDI/VDE 2000b). Inside the polymer material (thermoplastic, duromer or elastomer) surrounding the indenter during loading either fully elastic (elastomers), viscoelastic-plastic (such as polyethylene) or predominantly plastic (such as acrylonitrilebutadiene-styrene terpolymer) deformations are observed. Consequently, testing of plastics has some peculiarities enhanced by the same influencing factors like metals (Table 2).

Recent trends in materials research, process optimization and component development using nanostructured polymers such as block copolymers and polymer nanocomposites, which are often only available with small volume of samples, demand advanced testing procedures. In this context, modern indentation methods are very advantageous compared with other approaches for characterising the mechanical performance and the fracture safety of these materials, structures and components.

\section{Methodology \\ Materials and sample preparation TPU/POSS nanocomposites}

In this study, TPU/POSS nanocomposite materials (TPU - thermoplastic polyurethane, POSS - polyhedral oligomeric silsesquioxanes) have been investigated where the average molecular weight $M_{w}$ of polyethylene glycol (PEG) was varied from 10 to 35 $\mathrm{kg} / \mathrm{mol}$ and the PEG/POSS mole ratio from 3 to 12 . Some information about material preparation, molecular architecture and morphological design of a similar system are discussed in the literature (Wu et al. 2004). The samples were prepared in plate-like form having a thickness of ahout $0.35 \mathrm{~mm}$.

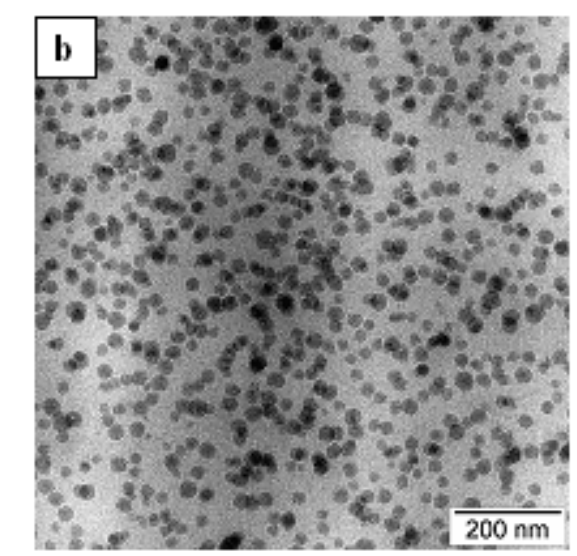

Fig. 1. TEM micrographs of thin sections of (a) a TPU/POSS nanocomposite (stained with $\mathrm{RuO}_{4}$; darker and lighter zones: hard and soft domains of TPU, black spots: POSS nanocrystals) and (b) a $\mathrm{PMMM} / \mathrm{SiO}_{2}$ nanocomposite (without staining).

Different microphase separation processes during sample preparation result in the observed microstructure where the TPU matrix is formed from hard ((35-50 $\mathrm{nm}$ in length and 10-15 $\mathrm{nm}$ in width, measured using transmission electron microscopy TEM) and soft phase domains; and POSS forms small crystalline aggregates only $5 \mathrm{~nm}$ in size (TEM)) which are relatively dispersed homogenously inside the hard phase (Fig. 1a). The hard phase and surrounding soft phase zones are locally ordered with a long period of 30-35 nm (as measured by TEM) or $31 \mathrm{~nm}$ (as measured by small-angle X-ray scattering SAXS, not shown), respectively. In the wide-angle X-ray scattering (WAXS) diagrams (not shown) several Bragg reflexes can be clearly indicated, which result from the coexistence of two different crystal species in the PEG/ POSS thermoplastic polyurethanes: the POSS crystallites having a rhombohedral (or equivalent hexagonal) unit cell with a less perfect crystalline structure and the PEG crystallites in the semicrystalline soft phase. From differential scanning calorimetry (DSC, not shown) it has been found that both POSS 
and PEG are hindering each other to crystallise (detailed information about the structure of the TPU/ POSS nanocomposites is discussed in the literature (Lach et al. 2010).

\section{PMMA/SiO ${ }_{2}$ nanocomposites}

The materials used in the form of solution-cast plates of about $3 \mathrm{~mm}$ thickness with a maximum edge length of $40 \mathrm{~mm}$ were polymethyl methacrylate (PMMA)/ $\mathrm{SiO}_{2}$ nanocomposites with $0-20$ wt.- $\% \mathrm{SiO}_{2}$. The composites were prepared using solution blending technique by Roehm GmbH \& Co. KG as described in the literature (Caretenute et al. 1995). The nanoparticles, $26 \mathrm{~nm}$ in average size (quantitatively analysed from TEM micrographs, Fig. 1b), were uniformly dispersed in the PMMA matrix without any evidence of agglomerates. As a consequence, the nanocomposites revealed excellent optical properties up to 20 wt.-\% of filler loading. The interparticle distance (ID) was computed to be $32 \mathrm{~nm}$ and $19 \mathrm{~nm}$ for the composites comprising 10 wt.-\% and 20 wt.-\% of $\mathrm{SiO}_{2}$ nanoparticles Lach et al. 2006).

\section{Equipment and methods}

Hardness measurement

The hardness and the elastic properties of all nanocomposites were determined by a recording microhardness tester (Fischerscope H 100C XYp) equipped with a Vickers diamond indenter at room temperature. Load $(F)$ versus indentation depth $(h)$ diagrams were measured at different locations on the samples up to a maximum load $F_{\max }$ of $1 \mathrm{~N}$ with a constant small loading rate of $50 \mathrm{mN} / \mathrm{s}$ similar to the unloading rate.

For small values of the indentation depth $h<6 \mu \mathrm{m}$ (i.e., micro range of load, $F<2 \mathrm{~N}$ ), the Martens hardness $H M$ defined in ISO 14577-1 (ISO Standard 2002) has to be corrected due to the erroneous indenter-tip configuration, i.e., the tip is never infinitely sharp but blunt in the micro or nano scale and the triangle faces of the Vickers pyramid do not converge to one point but form a small edge. Furthermore, the indenter tip acts as stress concentrator for the material below the tip especially for small indentation depths which results in increasing hardness for decreasing indentation depth. This has to be considered by a calibration procedure based on investigations of standard materials such as silicon, PMMA or polypropylene.
The above technique of indenter size correction and hardness calibration strongly depends on the quality of the determination of the calibration factor and is difficult to handle for layered structures. Therefore, another methods, to measure the hardness in the micro and nano range with high accuracy and also to be available on materials having a soft surface layer, will be introduced here which are more comfortable than the widely used calibration procedure. Based on the experimental observation, that Meyer's exponent is close to 2, a second-order Taylor series approximation for the load, $F(h)=F_{2}+F_{1}=a_{2} h^{2}+a_{1} h(F$ in $\mathrm{N}, h$ in $\mathrm{mm}$ ), can be used considering defects of the indenter and unavoidable stress concentrations in the material (Fröhlich et al. 1977), where $a_{1}$ and $a_{2}$ are parameters of the Taylor series. Now, the so-called Vickers hardness under load, $L_{2} V H=a_{2} / 26.43$, can be easily derived, which is identical to $H M$ in the macro range of load for homogeneous and isotropic materials.

Elastic properties, i.e., the values of the indentation modulus $E /\left(1-v^{2}\right)$ were determined by the procedure described in ISO 14577-1. For the TPU/POSS nanocomposites, the Poisson's ratio $v$ is found to be ranging close to $v=0.35$.

Indentation fracture mechanics

As shown in some investigations on polymer materials (mostly homopolymers) cracks formed in the vicinity of hardness impressions can be analysed within the scope of indentation fracture mechanics and can be used to determine the fracture toughness of these materials (Koch 2005, Lach et al. 2007, 2006. Based on a theoretical equation (Munz \& Fett 1999) found for the stress intensity factor $K_{\mathrm{I}}$ for cracks near a Vickers impression, altogether 19 approaches of indentation fracture mechanics have been developed which are applicable either for semielliptical or Palmqvist cracks or for both under specific conditions (Table 3; Lach et al. 2007, Ponton \& Rawlings 1989). In this paper, the approach of Laugier (1987) for Palmqvist cracks configuration was used, implying Eq. (1) to determine the stress intensity factor:

$K_{\mathrm{I}}=0.015 \cdot\left(\frac{c}{a}-1\right)^{-1 / 2} \cdot\left(\frac{E}{H V}\right)^{2 / 3} \cdot \frac{F}{c^{3 / 2}}$

Vickers hardness for polymers (in N/mm²), $a$ the half length of the impression diagonal (in $\mathrm{mm}$ ) and $c$ the radial crack length (in $\mathrm{mm}$ ). 
Here, $E$ is the modulus of elasticity (in MPa), $H V$ the Vickers hardness for polymers (in $\mathrm{N} / \mathrm{mm}^{2}$ ), $a$ the half length of the impression diagonal (in $\mathrm{mm}$ ) and $c$ the radial crack length (in $\mathrm{mm}$ ).

Tab le 3. Classification of indentation cracks.

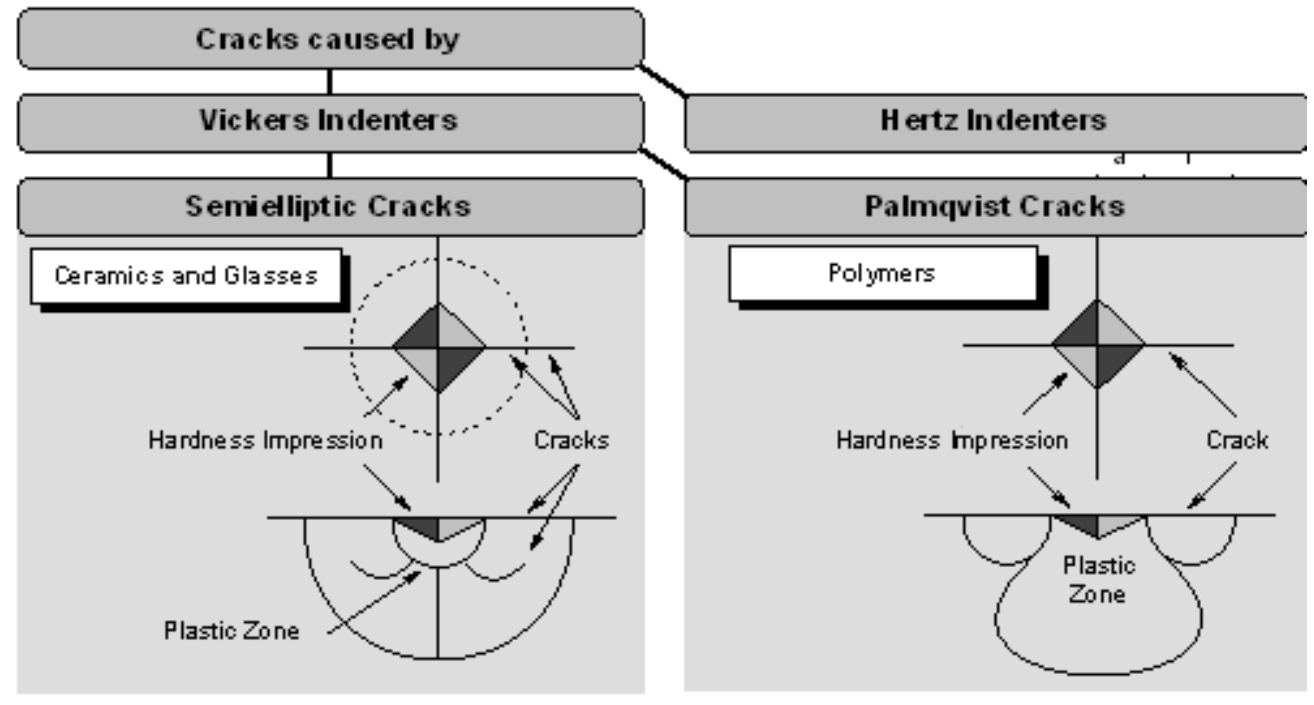

For glassy polymers such as PMMA und polystyrene (PS), it has been demonstrated that the Eq. (1) is not only of particular importance for ceramics (Laugier 1987) but also for brittle polymers (Lach et al. 2007, Koch 2005, Lach et al. 2009, Schöne et al 2011, O’Brien \& Parquette 2012) and other brittle organic substances (Lach et al. 2009). Indentation fracture mechanics approaches were not only successfully applied on PMMA based nanocomposites (this study) but also on other brittle thermoplastic nanocomposites such as $\mathrm{PS} / \mathrm{Al}_{2} \mathrm{O}_{3}$ and $\mathrm{PS} / \mathrm{AlOOH}$ nanocomposites (using $\mathrm{Al}_{2} \mathrm{O}_{3}$ nanorods and $\mathrm{AlOOH}$ nanorods; see Lach et al. 2009). Lach et al. 2007 analysed the fracture toughness $K_{\text {Ic }}$ (critical stress intensity factor) of two homopolymers (PMMA and PS) based both on the indentation fracture mechanics approach used in this study and a conventional technique applying singleedge-notched bend (SENB) specimens under fourpoint bending loading. Within the scatter of the data they found the same $K_{\mathrm{Ic}}$ values independently of the methods used as it has been already shown for ceramics by Laugier (1987).

\section{Results and Discussion}

Mechanical properties of TPU/POSS nanocomposites

For materials with hard bulk inside and soft surface layers or in the case of high surface roughness the original procedure without calibration to determine the hardness $(H M)$ leads to misleading results as shown in Fig. 2a. $H M$ at maximum load varies over one order of magnitude between $4 \mathrm{~N} / \mathrm{mm}^{2}$ and $23 \mathrm{~N} / \mathrm{mm}^{2}$ depending on the position on the plate due to position-dependent "soft layer" ranging from $20 \mu \mathrm{m}$ to $80 \mu \mathrm{m}$. In contrast, $L_{2} V H$ clearly shows the bulksoft layer structure which results from the preparation procedure of TPU/POSS with $L_{2} V H=27 \pm 5 \mathrm{~N} / \mathrm{mm}^{2}$ for the bulk material (Fig. 2b).

The hardness and the elastic properties are plotted as a function of PEG/POSS ratio and $M_{w}$ of PEG in Fig. 3. Most materials investigated showed an indentation behaviour with identical values of $L_{2} V H\left(\sim 26 \mathrm{~N} / \mathrm{mm}^{2}\right)$ and $E /\left(1-v^{2}\right)$ ( 450 MPa) except two having mechanical properties significantly improved compared to all other $\left(L_{2} V H=45 \mathrm{~N} / \mathrm{mm}^{2}\right.$ and $60 \mathrm{~N} / \mathrm{mm}^{2}, E /\left(1-v^{2}\right)=800 \mathrm{MPa}$ and $1240 \mathrm{MPa}$, respectively).

The reasons given by Fu et al. (2000) to explain the increase in elastic modulus as a function of POSS fraction cannot be confirmed by the results of the present study. They believe that, besides possible miscibility effects on stiffness caused by POSS modification, the POSS molecules attached to the hard segments or the POSS nanocrystals formed at high POSS fraction enhance the stiffness of the modified 
domains at the molecular or nanoscopic level, respectively. As a result, the macroscopic modulus is increased too. But the mechanical properties of most nanocomposites used here, except for two, are both independent on POSS fraction in principle and not influenced by the crystallinity of POSS. Thus, the reasons underlying an enhancement of the properties may be more complex. One can speculate about

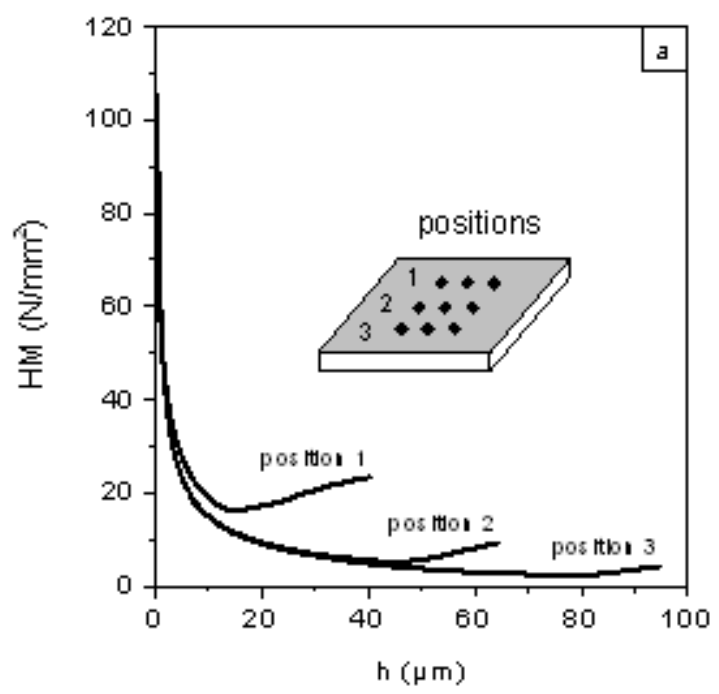

formation of a secondary POSS-polymer network, but a single reason why POSS is able to strengthen these materials as observed cannot be given now. Due to controversial results in this work and one by Fu et al. (2000), further investigations are essential in future to understand the relationships between mechanical properties and the microstructure of TPU/POSS nanocomposites in more detail.

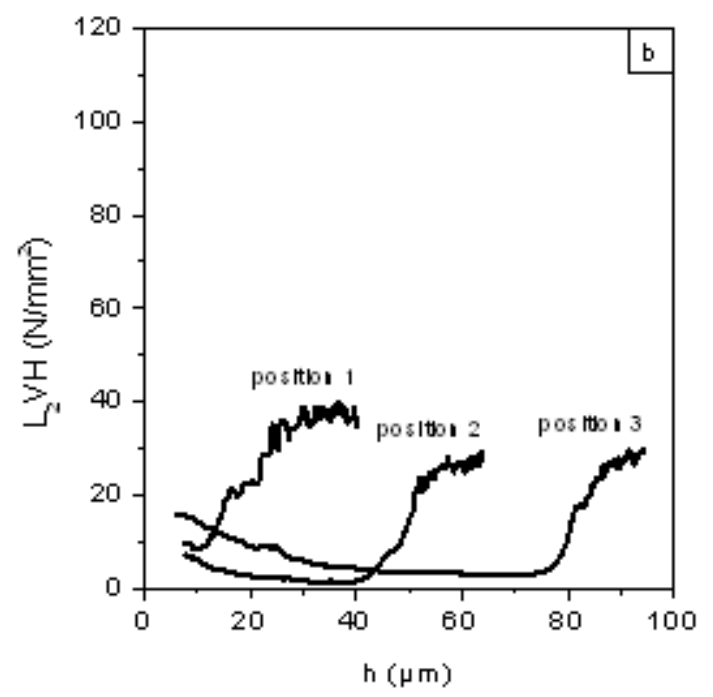

Fig. 2. Comparison of the hardness values $H M$ and $L_{2} V H$ for a TPU/POSS nanocomposite ( $M_{\mathrm{w}}$ of $P E G: 35$ $\mathrm{kg} / \mathrm{m}$ ol, $P E G / P O S S$ mol ratio: 12 ).

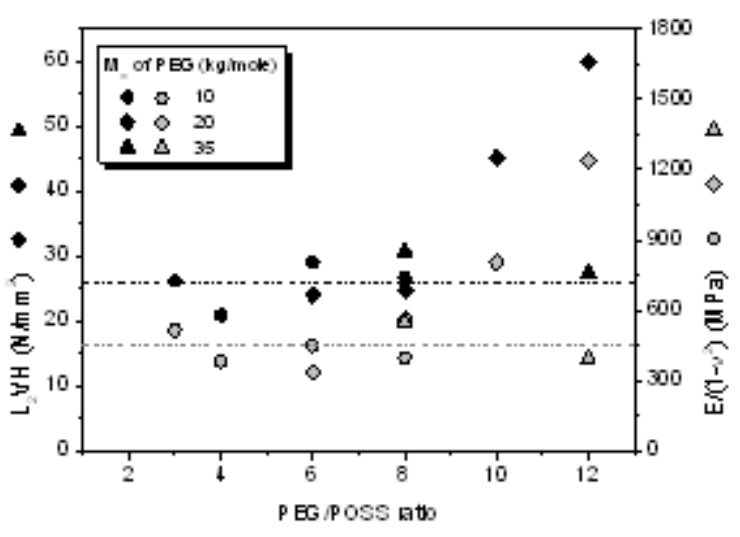

Fig. 3. Hardness $L_{2} V H$ and indentation modulus $E /\left(1-v^{2}\right)$ for the TPU/POSS nanocomposites as a function of $M_{\mathrm{w}}$ of PEG and PEG/POSS mole ratio

Fracture behaviour of PMMA/SiO ${ }_{2}$ nanocomposites In contrast to pure PMMA, the R-curve effect, enhancement in crack resistance (R) as a function of crack size (Lach et al. 2006), was not observed in the

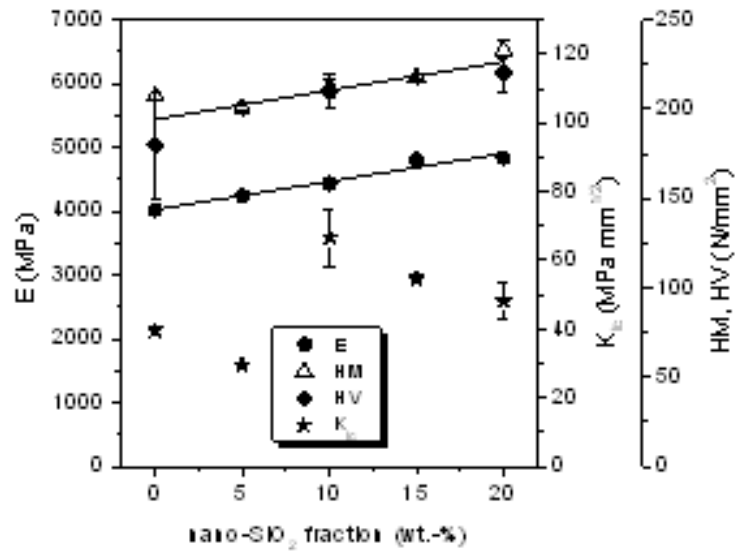

Fig. 4. Indentation modulus $E$, hardness $(H M, H V)$ and fracture toughness $K_{\text {Ic }}$ for the $\mathrm{PMMA} / \mathrm{SiO}_{2}$ nanocomposites as a function of $\mathrm{SiO}_{2}$ weight fraction

nanocomposites investigated. The fracture toughness $K_{\text {Ic }}$ was found to be dependent on the content of $\mathrm{SiO}_{2}$ nanoparticles, of which maximum value was observed at 10 wt.-\% (Fig. 4). A significant reduction in fracture 
toughness occurred at 20 wt.- $\% \mathrm{SiO}_{2}$ nanoparticles, which is associated with a percolation of the bound layers (interfacial layers) around the $\mathrm{SiO}_{2}$ particles. From DSC data, the thickness of the interfacial polymer layer was estimated to be about $9 \mathrm{~nm}$ (for more information see Lach et al. 2006) which is in the order of radius of gyration of the molecules (Katsikis 2008). In situ mechanical investigations of thin films using transmission electron microscopy (not shown) confirm the semiductile-to-brittle transition.

Compared to the fracture toughness, both the hardness ( $H M$ \& $H V$ ) and indentation modulus $E$ linearly increase as the $\mathrm{SiO}_{2}$ loading in the composite increases (Fig. 4).

\section{Acknowledgements}

For preparation of the TPU/POSS and PMMA/SiO nanocomposites, the authors thank Prof. Mather (Case Western Reserve University, Cleveland, USA) and Roehm GmbH \& Co. KG (Darmstadt, Germany), now Evonik Industries AG, particularly Dr. Albrecht and Dr. Goldacker.

\section{References}

Balta Calleja, F.J. and S. Fakirow. 2000. Microhardness of polymers. Cambridge Solid State Science Series, University Press, Cambridge, UK.

Carotenuto, G.L., Nicolais, X. Kuang and Z. Zhu. 1995. A method for the preparation of $\mathrm{PMMA}-\mathrm{SiO}_{2}$ nanocomposites with high homogeneity. Applied Composite Materials 2: 385-393.

Fröhlich, F., P. Grau and W. Grellmann. 1977. Performance and analysis of recording microhardness tests. Physica Status Solid: Applied Research A42: 79-89.

Fu, B. X., W. Zhang, B. S. Hsiao, M. Rafailovich, J. Sokolov, G. Johansson, B.B. Sauer, S. Phillips and R. Balnski. 2000. Synthesis and characterization of segmented polyurethanes containing polyhedral oligomeric silsesquioxanes nanostructured molecules. High Performance Polymers 12: 565-571.

Grellmann, W. and S. Seidler (Eds.) 2007. Polymer testing. Hanser, Munich, Germany.

Herrmann, K. 2007. Härteprüfung an Metallen und Kunststoffen: Grundlagen und Überblick zu modernen Verfahren. Expert Verlag, Renningen, Germany.

ISO 14577-1. 2002. metallic Materials - Instrumented indentation test for Hardness and Materials Parameters - Part 1: Test Method.

Katsikis, N. 2008. Particle-Polymer Interactions in Melts of Nano- and Microcomposites with Poly(methyl methacrylate) as Matrix. Ph.D. Thesis, University Erlangen-Nürnberg, Erlangen, Germany.

Koch, T. 2005. Morphologie und Mikrohärte von Polypropylen-Werkstoffen, Mensch \& Buch Verlag, Berlin, Germany.

Lach, R., G.M. Kim, G.H. Michler, W Grellmann and K. Albrecht. 2006. Indentation fracture mechanics for toughness assessment of $\mathrm{PMMA} / \mathrm{SiO}_{2}$ nanocomposites. Macromolecular Materials and Engineering, 291: 263-271.

Lach, R., L.A. Gyurova and W. Grellmann. 2007. Application of indentation fracture mechanics approach for determination of fracture toughness of brittle polymer systems. Polymer Testing 26:51-59.

Lach, R., T. Koch, B. Feichtenschlager, G. Kickelbick, W. Grellmann and S. Seidler. 2009. Application of improved indentation fracture mechanics approaches on in-situ polymerizied organic-inorganic nanocomposites. In: Proceedings of $12^{\text {th }}$ International Conference on Fracture, Ottawa, Canada, 8 pp.

Lach, R., G.H. Michler and W. Grellmann. 2010. Microstructure and indentation behaviour of polyhedral oligomeric silsesquioxanes-modified thermoplastic polyurethane nanocomposites. Macromolecular Materials and Engineering 295: 484-491.

Laugier, M. T. 1987. New formula for indentation toughness in ceramics. Journal of Materials Science Letters 8: 355356.

Munz, D. and T. Fett. 1999. Ceramics-Mechanical properties, failure Behaviour, Materials Selection. Springer, Berlin, Germany.

O’Brien, D. J. and B. Parquette. 2012. Polymer toughness transfer in a transparent interpenetrating glass-polymer composite. Composites Science and Technology, accepted for publication.

Ponton, C.B. and R.D. Rawlings. 1989. Vickers indentation fracture toughness test, Part 1: Review of literature and formulation of standardised indentation toughness equations. Materials Science and Technologies 5: 865-872.

Schöne, J., S. Arndt, C. Bierögel, R. Lach and W. Grellmann. 2011. Temperature dependent macrohardness - Method to characterise the creep and relaxation behaviour of polymers. In: Proceedings of $13^{\text {th }}$ Problemseminar Deformation und Bruchverhalten von Kunststoffen, Merseburg, Germany, 8 pp.

VDI/VDE 2616, Part 1. 2000a. Hardness testing of ,metallic materials, Beuth Verlag, Berlin, Germany.

VDI/VDE 2616, Part 2. 2000b. Hardness testing of plastics and rubber, Beuth Verlag, Berlin, Germany.

Wu, J., Q. Ge, K.A. Burke and P.T. Mather. 2004. Crystallization of POSS in a PEG-based multiblock polyurethane architecture: Toward a hybrid hydrogel. Materials Research Society Proceedings 847: EE10.2. 
Nepal Journal of Science and Technology Vol. 13, No. 2 (2012) 115-122 